\title{
On opulence driven poverty traps *
}

\author{
Charles van Marrewijk ${ }^{1}$ and Jos Verbeek ${ }^{2}$ \\ Erasmus University Rotterdam, Department of Economics, H8-16, P.O. Box 1738, \\ 3000 DR Rotterdam, The Netherlands \\ 2 Tinbergen Institute, Erasmus University, P.O. Box 1738, 3000 DR Rotterdam, The Netherlands \\ and The World Bank, 1818 H Street, N.W., Washington, DC 20433, USA
}

Received June 25, 1991 / Accepted April 1, 1992

\begin{abstract}
Endogenous population growth, i.e., making the rate of population growth dependent on society's opulence, causes parametric changes to have a larger impact and can cause multiplicity of steady states in a dynamic intertemporal optimization framework. This provides a simple explanation for the possibility of differing growth paths between countries (using a standard production function) or another explanation of the 'poverty trap'. We give two examples ('opulence sensitivity' and 'production sensitivity') that both give rise to three steady states in which poor (rich) countries will evolve over time to the low (high) income steady state. In both examples there are middle income countries that will choose the low (high) income steady state if they are impatient (patient), where patience is measured through the rate of time preference $\varrho$. Foreign aid in the form of a large transfer of capital from abroad enables poor and impatient middle income countries to move to the high income steady state.
\end{abstract}

\section{Introduction}

It is an obvious fact of this century that poor countries tend to have higher rates of population growth combined with low levels of per capita income, as emphasized by Cigno (1992), see also Ehrlich (1990) and Rosenzweig (1990). ${ }^{1}$ This leads to a negative relationship between per capita income and fertility. A relationship that can be found in aggregate data, whether examined cross-sectionally

\footnotetext{
* We would like to thank Nico Heering, Martijn Herrmann, Theo Junius, Ngo van Long, Ad Pikkemaat, John Pitchford, Lakshmi Raut, Casper de Vries, an anonymous referee, participants of workshops at the University of Groningen and the University of Amsterdam and especially Harry van Dalen for valuable comments. The article was presented at the fifth annual conference of the European Society of Population Economics, Pisa 1991. The views expressed in the article are those of the authors and not necessarily those of the World Bank.

1 Recent microeconomic studies stress also the importance of the quality of children, see e.g. Barro and Becker (1939) or Raut (1991).
} 
or over time. ${ }^{2}$ We opt in this study to endogenize the growth rate of the population in such a way that it stresses the empirically supported negative relation between fertility and per capita income. Fertility declines as per capita income rises. There are in principal three ways to endogenize the fertility decision (see Pitchford 1974): Arithmetical theories, theories of demographic transition and the so-called Ricardian approach of population growth. ${ }^{3}$ We embed the first approach in a standard Ramsey-model of infinitely lived agents (which is equivalent to a dynastic overlapping generations model, with fully operative bequests).

Recent economic growth theory, see e.g. Romer (1986a) or Lucas (1988), shows that growth paths between developed and less developed countries differ if there are externalities in production. Earlier work by Skiba (1978), arrived at the same conclusion by positing a convexo-concave production function. One of the interesting features of our model is that we derive the same result using a standard neoclassical production function. We arrive at this conclusion through the possibility of multiple steady states (alternatingly saddlepoint stable and unstable), see also Nelson (1956), where the initial capital-labor ratio determines whether the economy will end up in a high per capita income/low fertility steady state or in a low per capita income/high fertility steady state. The less developed economy can get stuck in a variant of the 'Malthusian trap', also called the poverty trap. The only way out of the poverty trap is through an injection of capital (a large transfer) from abroad. Our model therefore, supports Tinbergen's view that aid from the developed countries to the less developed countries has to be increased dramatically (Tinbergen wants foreign aid to be quadrupled) to overcome the poverty trap.

Section 2 introduces the model, Sect. 3 analyzes the steady state(s), Sect. 4 discusses stability, Sect. 5 gives two illuminating examples to identify the various causes of multiplicity, and finally, Sect. 6 concludes.

\section{The model}

In order to explore the dynamics of endogenous population growth, we use the Ramsey infinite horizon model. Output $[Y(t)]$ in period $t$ is produced using capital $[K(t)]$ and labor $[L(t)]$. The concave production function $(F)$ exhibits constant returns to scale. Romer (1986a) argues that the economy should be characterized by an increasing returns to scale production function. However, recent empirical evidence, see Benhabib and Jovanovic (1991) and Mankiw et al. (1992), does not support this view. We, therefore, opt for constant returns to scale. In per capita form:

\footnotetext{
2 A simple regression using United Nations data for the population growth rate $y$ (1975-1980 average) and World Bank data for per capita purchasing power parity income levels $z$ (1975) leads to: $y=2.8896-0.0273 z$, with $R^{2}=0.59$ and $t$-values in parentheses. Countries with less than 1 million (20.9) $\quad(-10.6)$

inhabitants were not included, with 20-50 million inhabitants were counted twice, with 50-150 million inhabitants were counted three times and with more than 150 million inhabitants four times. The paper by Sato and Davis (1971), which is frequently referred to, postulates a positive relation between population growth and per capita income because the death rate is lower in rich countries. The empirical evidence does not support their view.

3 Our model can easily generate a theory of demographic transition as a special case, see Sect. 4. The Ricardian theory has been refuted by the empirical evidence as argued above.
} 


$$
y(t)=f[k(t)] .
$$

One can use output either to invest $[i(t)]$ or to consume $[c(t)]$.

$$
y(t)=c(t)+i(t) .
$$

The objective of the economy (its government, a 'omniscient central planner', etc.) is to maximize instantaneous per capita utility $U[c(t)]$ over an infinite time horizon using the time preference rate, or the subjective discount rate, $\varrho$, which is assumed to be constant and strictly positive.

$$
\max _{c} \int_{0}^{\infty} \exp (-\varrho t) U[c(t)] \mathrm{d} t .
$$

We make the following familiar assumptions. ${ }^{4}$

$A$ l. $f[k(t)]$ is twice continuously differentiable with

$$
\begin{array}{ll}
f_{k}[k(t)]>0 & f_{k k}[k(t)]<0 \\
\lim _{k \downarrow 0} f_{k}[k(t)]=\infty & \lim _{k \uparrow \infty} f_{k}[k(t)]=0
\end{array}
$$

A2. $U[c(t)]$ is twice continuously differentiable with

$$
U_{c}[c(t)]>0 \quad U_{c c}[c(t)]<0 \quad \lim _{c \downarrow 0} U_{c}[c(t)]=\infty .
$$

The speed of population growth reacts to the prosperity level. In the sequel time derivatives will be denoted by placing a dot over the variable.

$$
\dot{L}(t) / L(t)=g(\cdot) .
$$

We distinguish between two basic specifications of the function $g(\cdot)$. The prosperity level can be identified by the per capita level of consumption, assumption $A 3$ below, which emphasizes that it is the need to consume basic goods which causes the fertility problems. We will refer to this assumption as "consumption driven' population growth. Alternatively, it can be argued that the prosperity level should be identified by the per capita production level, $y(t)$, or the level of the wage rate, $w(t)$. Since in a competitive economy both the production level, $y(t)=f[k(t)]$, and the wage rate, $w(t)=f[k(t)]-k(t) f_{k}[k(t)]$, are increasing functions of the capital-labor ratio these specifications would make the speed of population growth a function of the capital-labor ratio, assumption $A 3^{\prime}$ below. We will refer to this assumption as 'production or wage driven' population growth. In short

A3. $g(\cdot)$ is a twice continuously differentiable function of $c(t)$ with

\footnotetext{
$4 \quad$ We will denote derivatives with a subindex, this means that if $g(z)$ is a function then its first derivative is $g_{z}(z)$ and its second derivative is $g_{z z}(z)$ etc.
} 


$$
\begin{array}{ll}
\lim _{c \downarrow 0} g[c(t)]=\theta>0 & \lim _{c \uparrow \infty} g[c(t)]=0 \\
g_{c}[c(t)]<0 & g_{c c}[c(t)]>0
\end{array}
$$

$A 3^{\prime} \cdot g(\cdot)$ is a twice continuously differentiable function of $k(t)$ with

$$
\begin{array}{ll}
\lim _{k \downarrow 0} g[k(t)]=\theta>0 & \lim _{k \uparrow \infty} g[k(t)]=0 \\
g_{k}[k(t)]<0 & g_{k k}[k(t)]>0
\end{array}
$$

Hence $A 3\left(A 3^{\prime}\right)$ assumes a negative relationship between population growth and per capita consumption (income or wage) on the aggregate level as is consistent with the empirical evidence. ${ }^{5}$ For a microeconomic justification of this relationship, based on individual decision making regarding fertility in an overlapping generations framework, the interested reader is referred to Raut (1991). In the sequel we will restrict attention to consumption driven population growth, assumption $A 3$. All conclusions (with a few minor exceptions) carry over to the case of production or wage driven population growth, assumption $A 3^{\prime}$, see the appendix.

The capital stock increases due to gross investment and decreases due to depreciation $[\mu K(t)]$

$$
k(t)=f[k(t)]-\mu k(t)-g[c(t)] k(t)-c(t) .
$$

Before solving the optimality problem we specify one more (technical) assumption and define the following elasticities ${ }^{6}$

$$
\begin{aligned}
& \sigma_{U_{c}}(c) \equiv-\left[\mathrm{d} U_{c}(c) / \mathrm{d} c\right]\left[c / U_{c}(c)\right]=-c U_{c c}(c) / U_{c}(c)>0 \\
& \sigma_{I_{c}}(c, k) \equiv[\partial(\partial \dot{k} / \partial c) / \partial c][(\partial \dot{k} / \partial c)]=c k g_{c c}(c) /\left[1+k g_{c}(c)\right]>0 \\
& \sigma_{I_{k}}(c, k) \equiv-[\partial(\partial \dot{k} / \partial c) / \partial k][k /(\partial \dot{k} / \partial c)]=-k g_{c}(c) /\left[1+k g_{c}(c)\right]>0 .
\end{aligned}
$$

Hence, $\sigma_{U_{c}}(c)$ equals (minus) the elasticity of marginal utility, which is a local measure of the curvature of the utility function, $\sigma_{I_{c}}(c, k)$ is the elasticity of marginal per capita investment $\left[1+k g_{c}(c)\right]$ with respect to per capita consumption, which can then be seen as the curvature of the per capita investment function with respect to per capita consumption. The same holds for $\sigma_{I_{k}}(c, k)$ with respect to per capita capital and investment. Define $\mathscr{D} \equiv\left\{(k, c) \mid 1+k g_{c}(c) \geq 0\right\}$, then the final assumption is

A4. $(k, c) \in \mathscr{D}$.

Assumption $A 4$ basically implies that at high capital-labor ratios, and hence high levels of per capita production, the consumption level will not be too small (see Figs. 3 and 4 below). It can be justified as follows: ${ }^{7} g(c) k$ is the capital per head needed to equip the increment in population with the same capital as the existing

\footnotetext{
5 It might be argued that the population growth function should have a point of inflexion, i.e. $g_{c c}$ is negative at first and positive for consumption above some value $\bar{c}$. This will be an additional source of multiplicity but requires some restrictions to establish existence of a solution. For ease of exposition we restrict attention to the case of convex $g(c)$.

6 The time index will be suppressed in the sequel unless confusion may arise.

7 We thank John Pitchford for this formulation.
} 
population. Suppose $c$ rises. If $\left[g_{c}(c) k+1\right]$ is negative it means that a rise in consumption produces such a fall in population growth and hence reduction in capital needed to equip new population that the residual output $f(k)-g(c) k-c$ actually rises! In practice this seems so unlikely that it can be assumed away.

The problem can be summarized as

\section{Problem P.}

$$
\begin{aligned}
& \max _{c} \int_{0}^{\infty} \exp (-\varrho t) U[c(t)] \mathrm{d} t \quad \text { subject to } \\
& \dot{k}(t)=f[k(t)]-\{\mu+g[c(t)]\} k(t)-c(t) ; \quad k(0) \text { given }(k, c) \in \mathscr{D} .
\end{aligned}
$$

Proposition 1. Under assumptions $A 1-A 4$ there exists a solution to problem $P$.

Proof. We can apply Romer's (1986b) theorem. He shows that maximization of $\int_{0}^{\infty} \exp (\varrho t) \mathscr{F}[k(t), \dot{k}(t)] \mathrm{d} t$ has a solution under two conditions. The first condition states that $\mathscr{F}$ should be concave in $\dot{k}$. The consumption level $c$ from (5) can be written as a function of $k$ and $k$ (using $A 4$ ) which can then be substituted in the function to be maximized. Under $A l-A 4$ we get

$$
\begin{aligned}
& \mathscr{F}_{k}=-U_{c}(c) /\left[1+k g_{c}(c)\right]<0 \\
& \mathscr{F}_{k k}=-U_{c}(c)\left[\sigma_{U_{c}}(c)+\sigma_{I_{c}}(c, k)\right] /\left[c\left(1+k g_{c}(c)\right)^{2}\right]<0 .
\end{aligned}
$$

Hence the first condition is fulfilled. The second condition in Romer's theorem is always fulfilled as the rate of population growth (through $A 3$ ) is bounded.

The optimal solution can be characterized using the maximum principle. Define the present-value Hamiltonian.

$$
H=[U(c)+\lambda[f(k)-[\mu+g(c)] k-c] \exp (-\varrho t) .
$$

Necessary conditions for an optimal trajectory of $k(t), c(t)$ and $\lambda(t)$ are [Romer (1986b) and Blanchard and Fisher (1989)]

$$
\begin{aligned}
& U_{c}(c)-\lambda\left[k g_{c}(c)+1\right]=0 \\
& \dot{\lambda}=\lambda\left[\varrho+\mu+g(c)-f_{k}(k)\right] \\
& \lim _{t \rightarrow \infty} k(t) \lambda(t) \exp (-\varrho t)=0
\end{aligned}
$$

Naturally, (7) only holds for $(k, c) \in \operatorname{int}(\mathscr{D})$, otherwise, when this restriction is binding, $c$ solves $1+k g_{c}(c)=0$. Differentiate Eq. (7) with respect to time and eliminate $\lambda$ and $\dot{\lambda}$ by using (5), (7) and (8) to get the following system of differential equations

$$
\begin{aligned}
\dot{c} & =\left\{c /\left[\sigma_{U_{c}}(c)+\sigma_{I_{c}}(c, k)\right]\right\}\left\{f_{k}(k)+\sigma_{I_{k}}(c, k)(\dot{k} / k)-[\varrho+\mu+g(c)]\right\} \\
\dot{k} & =f(k)-[\mu+g(c)] k-c .
\end{aligned}
$$




\section{Steady state values}

The steady state values of system (10) are given by $\dot{c}=\dot{k}=0$. Denote the steady state by $c^{*}$ and $k^{*}$, then ${ }^{8}$

$$
\begin{aligned}
& f_{k}\left(k^{*}\right)-\left[\varrho+\mu+g\left(c^{*}\right)\right]=0 \\
& f\left(k^{*}\right)-\left[\mu+g\left(c^{*}\right)\right] k^{*}-c^{*}=0 .
\end{aligned}
$$

The first equation in system (11) extends the modified neoclassical 'golden rule' of accumulation by taking the dependence of the population growth rate on the per capita level of consumption into consideration. The neoclassical dichotomy, in which the rate of time preference, the depreciation rate and the production function dictate the steady state level of capital independently of the consumption level, disappears. To examine the effect changes in the rate of time preference ${ }^{9}$ and the depreciation rate have on the steady state values of $c$ and $k$ differentiate

$$
\left[\begin{array}{cc}
f_{k k} & -g_{c} \\
\varrho & -\left(1+k^{*} g_{c}\right)
\end{array}\right]\left[\begin{array}{l}
\mathrm{d} k^{*} \\
\mathrm{~d} c^{*}
\end{array}\right]=\left[\begin{array}{c}
\mathrm{d} \varrho+\mathrm{d} \mu \\
k^{*} \mathrm{~d} \mu
\end{array}\right]
$$

and solve to get

$$
\begin{array}{ll}
\mathrm{d} k * / \mathrm{d} \mu=-1 / \Delta<0 & \mathrm{~d} k * / \mathrm{d} \varrho=-\left(1+k^{*} g_{c}\right) / \Delta<0 \\
\mathrm{~d} c * / \mathrm{d} \mu=-\left(\varrho-k^{*} f_{k k}\right) / \Delta<0 & \mathrm{~d} c * / \mathrm{d} \varrho=-\varrho / \Delta<0,
\end{array}
$$

where $\Delta \equiv-\left(1+k * g_{c}\right)\left(f_{k k}+\varrho \sigma_{I_{k}} / k\right)$ is the determinant of the Jacobian. If the equilibrium is locally stable then $\boldsymbol{\Delta}>0$ (see Sect. 4).

To investigate the effect of endogenization of the population growth rate we have to look at a 'comparable' exogenous economy. By this we mean an economy such that the exogenous growth rate of the population, $\pi$ say, is equal to the endogenous growth rate of the population in the steady state, i.e. $\pi=g\left(c^{*}\right) \cdot{ }^{10}$ It is straightforward to derive

$$
\begin{array}{ll}
\mathrm{d} k * /\left.\mathrm{d} \mu\right|_{\mathrm{ex}}=-1 / \Delta^{\prime}<0 & \mathrm{~d} k * /\left.\mathrm{d} \varrho\right|_{\mathrm{ex}}=-1 / \Delta^{\prime}<0 \\
\mathrm{~d} c * /\left.\mathrm{d} \mu\right|_{\mathrm{ex}}=-\left(\varrho-k^{*} f_{k k}\right) / \Delta^{\prime}<0 & \mathrm{~d} c * /\left.\mathrm{d} \varrho\right|_{\mathrm{ex}}=-\varrho / \Delta^{\prime}<0
\end{array}
$$

where $\Delta^{\prime}=-f_{k k}>\Delta$. Hence we see $\mathrm{d} k / \mathrm{d} \varrho<\mathrm{d} k /\left.\mathrm{d} \varrho\right|_{\mathrm{ex}}<0$, etc. and therefore arrive at Proposition 2.

Proposition 2. The introduction of consumption driven population growth causes changes in the rate of time preference and the depreciation rate to have a larger impact on the steady state levels of consumption and capital.

This effect is easy to understand. Suppose, for example, that the rate of time preference rises. In the exogenous case this affects the marginal productivity

8 As will become clear in Sect. 4 at least one but possibly more steady state(s) exist(s).

9 Not to be confused with endogenous time preference formation.

10 Naturally, for the exogenous economy we have $g_{c}=g_{c c}=0$. 
'golden rule' condition, the first equation of system (11), which determines the new capital level. This in turn determines how much can be consumed through the second equation of system (11). With endogenous population growth this dichotomy disappears. An increase in the rate of time preference initially reduces the capital level through the marginal productivity efficiency condition. This reduces the consumption level, which increases the growth rate of the population, which reduces the capital level through the marginal efficiency criterion, etc.

We note, furthermore, that a change in time preference or depreciation has the same effect on the level of capital in the exogenous economy, i.e. $\mathrm{d} k /\left.\mathrm{d} \varrho\right|_{\mathrm{ex}}=$ $\mathrm{d} k /\left.\mathrm{d} \mu\right|_{\mathrm{ex}}<0$, whereas that is not the case in the endogenous economy, where we have $\mathrm{d} k / \mathrm{d} \varrho<\mathrm{d} k / \mathrm{d} \mu<0$. This is because the capital level in the exogenous case is purely determined by the marginal productivity condition, the first equation of system (11). A change in $\varrho$ or $\mu$ identically affects this equation. An increase in depreciation, however, reduces the amount that can be consumed even further, see the second equation of system (11), as compared to an increase in time preference due to additional depreciation, the term $\mu k *$ The extra decrease in consumption causes an extra increase in population growth and therefore an extra decrease in the capital stock.

\section{Stability and multiplicity}

Recall Eq. (11)

$$
\begin{aligned}
& f_{k}\left(k^{*}\right)-\left[\varrho+\mu+g\left(c^{*}\right)\right]=0 \\
& f\left(k^{*}\right)-\left[\mu+g\left(c^{*}\right)\right] k^{*}-c^{*}=0 .
\end{aligned}
$$

At a steady state we have $k=0$ and $\dot{c}=0$, hence we can write $c$, using the first equation of (11), as a function of $k$

$$
c=g^{-1}\left[f_{k}(k)-(\varrho+\mu)\right] \equiv R(k) .
$$

Let $q \equiv f_{k}-(\varrho+\mu)$, then $g_{q}^{-1}<0$ and $g_{q q}^{-1}>0$. Using the fact that in a steady state $g(c)=f_{k}-(\varrho+\mu)$, we can write the second equation of (11) also as a function of $k$

$$
c=f(k)-k f_{k}(k)+\varrho k \equiv P(k) .
$$

For a steady state we need $P(k)=R(k)$, which will usually not, as will become clear in the sequel, result in an unique steady state. Careful investigation of the functions $P$ and $R$ give us the following information

$$
\begin{array}{ll}
P_{k}(k)=-k f_{k k}+\varrho>0 & R_{k}(k)=g_{q}^{-1} f_{k k}>0 \\
P_{k k}(k)=-f_{k k}-k f_{k k k} & R_{k k}(k)=g_{q q}^{-1} f_{k k}^{2}+g_{q}^{-1} f_{k k k} \\
P(0) \geq 0 & R(\tilde{k})=0 \quad \text { for } \tilde{k}=f_{k}^{-1}(\theta+\varrho+\mu) \\
P(\infty)=\infty & R(\hat{k})=\infty \quad \text { for } \tilde{k}=f_{k}^{-1}(\varrho+\mu) .
\end{array}
$$


Note, first of all, that $R(k)$ is not defined for $k<\tilde{k}$ and $k \geq \hat{k}$, hence steady states can only be found in the region between $\tilde{k}$ and $\hat{k}$. Secondly, the curvature (convexity and/or concavity) of the functions $R$ and $P$ depends on the third derivative of the production function, about which the neoclassical theory of production tells us nothing. For the important class of CES production functions, however, $f_{k k k}$ is positive for all $k$ if and only if the elasticity of substitution, $\sigma$, is bigger than or equal to $1 / 2$, while $P(k)$ is concave if and only if the elasticity of substitution is bigger than or equal to 1 . Both functions are continuous, however, and we can apply the mean value theorem to conclude that there exists at least one steady state.

If we linearize system (10) around the steady state and use (11) we get

$$
\left[\begin{array}{l}
\dot{c} \\
\dot{k}
\end{array}\right]=\left[\begin{array}{cc}
0 & \omega\left(f_{k k}+\varrho \sigma_{I_{k}} / k\right) \\
-\left(1+k g_{c}\right) & \varrho
\end{array}\right]\left[\begin{array}{l}
c-c^{*} \\
k-k^{*}
\end{array}\right]
$$

with $\omega \equiv c /\left[\sigma_{U_{c}}(c)+\sigma_{I_{c}}(c, k)\right]>0$.

The trace of the matrix is $\varrho$ and the determinant is $-\omega \Delta$. If the model is locally saddle point stable one eigenvector must be positive and one negative. This implies that the determinant must be negative, and hence $\Delta$, as defined in Sect. 3, must be positive. Hence a necessary and sufficient condition for a steady state to be saddle point stable is that $-\left(f_{k k}+\varrho \sigma_{I_{k}} k\right)\left(1+k g_{c}\right)>0$. Fill in the definition of $\sigma_{I_{k}}$ to get

$$
\begin{aligned}
& -\left[f_{k k}+k f_{k k} g_{c}-\varrho g_{c}\right]>0 \\
& \left(-g_{c}\right)\left[-f_{k k} / g_{c}-f_{k k} k+\varrho\right]<0 \\
& \left(-g_{c}\right)\left[P_{k}-R_{k}\right]<0 .
\end{aligned}
$$

Which means that the $R(k)$ curve must cut the $P(k)$ curve from below to have a saddle point stable stationary state. From the properties of the $R(k)$ and $P(k)$ curves discussed above it is clear that they intersect an odd number of times, while $R(k)$ cuts $P(k)$ from below the first time. We therefore can state Proposition 3.

Proposition 3. There is an odd number of steady states, alternating in local saddle-point stability, the first of which is locally saddle-point stable.

The possibility of the 'poverty trap' is then, in the presence of three steady states, easily illustrated in Figs. 1 and 2, while the dynamics are illustrated in Figs. 3 and 4 . There is a low income, high fertility steady state and a high income, low fertility steady state. The unstable steady state can be characterized by spirals, which are saddle paths leading to the low or high income steady state (see Fig. 4). If $k(0)$ is in between $\boldsymbol{A}$ and $\boldsymbol{B}$ it is not a priori clear which saddle path will be chosen. Because the value function is rising in $k(0)$, however, there is a unique cut-off point $k_{T}\left(\boldsymbol{T}\right.$ for trap) in between $\boldsymbol{A}$ and $\boldsymbol{B}$, such that for $k(0)<k_{T}$ the economy will move to the low income steady state, while for $k(0) \geq k_{T}$ the economy will move to the high income steady state, see Brock and Malliaris (1989, Chap. 6), Skiba (1978) and Dechert and Nishimura (1983). If the initial capitallabor ratio is 'low' (below $k_{T}$ ) then the economy is forced to the low income, high fertility steady state. If the initial capital-labor ratio is 'high' (above $k_{T}$ ), 
then the economy will reach the high income, low fertility steady state. Thus poor countries will reach different steady states than rich countries even if preferences and production functions are identical and do not exhibit 'peculiarities'. The possible causes of multiplicity will be examined in the next section.

The only way to escape from the poverty trap is through foreign aid in the form of a transfer of capital that raises the capital-labor ratio above the level $k_{T}$. Here it should be emphasized that 'piecemeal' aid from abroad to a poor country will not make it possible for that country to reach the high welfare steady state as long as the critical value $k_{T}$ is not reached. This would then argue, in contradistinction to Dellas and De Vries (1991), for a large once and for all capital donation from abroad.

\section{Two examples}

To illustrate Proposition 3 we will investigate two examples. The first example leads to a concave $P(k)$ function and a point of inflexion in the $R(k)$ function. The second example will have a convexo-concave $P(k)$ function, see Brock and Malliaris (1989, Chap. 6), while the $R(k)$ function will be convex. Most of the literature (see e.g. Liviatan and Samuelson 1969; Skiba 1978; Dechert and Nishimura 1983; Dellas and De Vries 1991) views "irregularities", i.e. non-differentiability or convexo-concavity, in the production function as the cause of multiplicity. In our framework this is an overly strong condition as a perfectly well-behaved concave production function can still be the cause of multiplicity as is illustrated in Example 2 below. In Example 1 multiplicity is caused by opulence sensitivity.



Fig. 1. Multiplicity through opulence sensitivity 
Example 1. For $g(c)$ we take a logistic type of population growth function, first introduced by the Dutch mathematical-biologist Verhulst in 1837 .

$$
g(c)=a-b Z(c), \text { where } Z(c)=a /[(a-b) \exp (-a c)+b] .
$$

This function will satisfy the posited properties of $g(c)$ if $(a-b) / b \leq 1$. Note that

$$
\begin{aligned}
& \lim _{c \rightarrow \infty} Z(c)=a / b \Rightarrow \lim _{c \rightarrow \infty} g(c)=0 \\
& \operatorname{Lim}_{c \rightarrow 0} Z(c)=1 \quad \Rightarrow \lim _{c \rightarrow 0} g(c)=a-b \\
& g_{c}=-b[Z(c)]^{2}(a-b) \exp (-a c)<0 \\
& g_{c c}=-b[Z(c)]^{3}(a-b) \exp (-a c)[(a-b) \exp (-a c)-b]>0 \quad \text { if }(a-b) / b \leq 1 \\
& g^{-1}(x)=(1 / a) \ln [(a-b)(a-x) / b x] \\
& R(k)=(1 / a) \ln \left\{(\boldsymbol{a}-\boldsymbol{b})\left[a+\varrho+\mu-f_{k}(k)\right] / b\left[f_{k}(k)-\varrho-\mu\right]\right\} .
\end{aligned}
$$

The production sector produces with a Cobb-Douglas technology $\left(y=A k^{\beta}\right.$ with $0<\beta<1$ ), which leads to the following properties of $P(k)$ and $R(k)$

$$
\begin{aligned}
P(k) & \equiv f(k)-k f_{k}(k)+\varrho k=A(1-\beta) k^{\beta}+\varrho k \\
P_{k}(k) & =-k f_{k k}+\varrho=A \beta(1-\beta) k^{\beta-1}+\varrho>0 \\
P_{k k}(k) & =-f_{k k}-k f_{k k k}=-A \beta(1-\beta)^{2} k^{\beta-2}<0 \\
R(k) & \equiv g^{-1}\left[f_{k}(k)-(\varrho+\mu)\right]=g^{-1}\left[\beta A k^{\beta-1}-(\varrho+\mu)\right] \\
& =(1 / a) \ln \left\{(a-b)\left[a+\varrho+\mu-\beta A k^{\beta-1}\right] / b\left[\beta A k^{\beta-1}-\varrho-\mu\right]\right\} \\
R_{k}(k) & =g_{q}^{-1} f_{k k}=-A \beta(1-\beta) k^{\beta-2} / g_{c}>0 \\
R_{k k}(k) & =g_{q q}^{-1} f_{k k}^{2}+g_{q}^{-1} f_{k k k} \\
& =g_{q q}^{-1}\left[A \beta(1-\beta) k^{\beta-2}\right]^{2}+g_{q}^{-1} A \beta(1-\beta)(2-\beta) k^{\beta-3} .
\end{aligned}
$$

Figure 1 illustrates the situation. It follows from Proposition 3 that the low per capita consumption steady state and the high per capita consumption steady state are saddlepoint stable. Clearly, the occurrence of multiplicity depends on the specific parameter setting. An example that gives rise to multiplicity, as can be readily checked on any personal computer, is the following: $a=2, b=1$ [hence $(\boldsymbol{a}-\boldsymbol{b}) / \boldsymbol{b}=1], A=1, \beta=\frac{1}{2}, \varrho=0.05$ and $\mu=0.05$. In this case $\left(k_{1}^{*}, k_{2}^{*}, k_{3}^{*}\right) \approx(0.4$, $5,24.4)$.

Example 2. Assume for $g(c)$ the following function

$$
g(c)=a-a[\exp (-b c)+1]^{-1} .
$$

Hence

$$
\begin{array}{ll}
g(0)=a / 2 & g(\infty)=0 \\
g^{-1}(q)=(1 / b) \ln [(a-q) / q] & \text { for } q \in(0, a / 2] .
\end{array}
$$




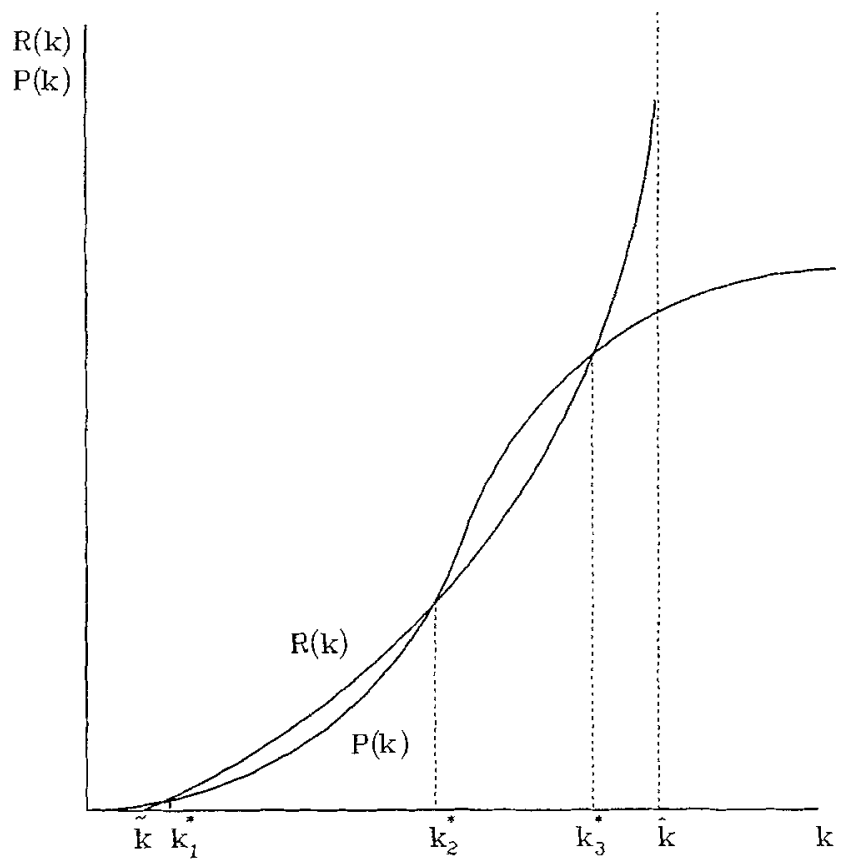

Fig. 2. Multiplicity through production sensitivity

The production technology used in this example is of the CES type

$$
f(k)=A\left[k^{\delta}+1\right]^{1 / \delta} \quad \delta \in(-\infty, 1] \backslash\{0\} .
$$

Which implies for the $P(k)$ function

$$
\begin{aligned}
& P(k)=A\left[k^{\delta}+1\right]^{[1 / \delta)-1]}+\varrho k \\
& P_{k}(k)=(1-\delta) \boldsymbol{A}\left[k^{\delta}+1\right]^{[(1 / \delta)-2]} k^{\delta-1}+\varrho>0 \\
& P_{k k}(k)=-(1-\delta) \boldsymbol{A}\left[k^{\delta}+1\right]^{[(1 / \delta)-3]} k^{\delta-2}\left[\delta k^{\delta}+(1-\delta)\right] .
\end{aligned}
$$

Note that $P_{k k}(k)<0$ if the elasticity of substitution, $\sigma \equiv 1 /(1-\delta)$, is bigger than or equal to one, which implies that $P(k)$ is concave. Whereas $P(k)$ is convexoconcave for $\sigma<1$, with the inflexion point at $\bar{k}=[-(1-\delta) / \delta]^{1 / \delta}$. The $R(k)$ function can be written as

$$
\begin{aligned}
R(k) \equiv & g^{-1}\left[f_{k}(k)-(\varrho+\mu)\right] \\
= & (1 / b) \ln \left\{\boldsymbol{a}-\boldsymbol{A}\left[k^{\delta}+1\right]^{[(1 / \delta)-1]} k^{\delta-1}\right. \\
& +\varrho+\mu\} /\left\{\left[\boldsymbol{A}\left[k^{\delta}+1\right]^{[(1 / \delta)-1]} k^{\delta-1}-\varrho-\mu\right\}\right] .
\end{aligned}
$$

For $\sigma<1$ we can then have an outcome as drawn in Fig. 2, where multiplicity is caused by production sensitivity, i.e. $P(k)$ is convexo-concave, whereas $R(k)$ is convex. Again, the occurrence of multiplicity depends on the specific parameter setting. As an example take $a=1.9, b=3.1, A=1, \delta=-4$ (hence $\sigma=1 / 5$ ), $\varrho=0.01$ and $\mu=0.01$. This gives rise to $\left(k_{1}^{*}, k_{2}^{*}, k_{3}^{*}\right) \approx(0.56,0.87,1.08)$. 


\section{Discussion}

A short discussion of the dynamics in $(k, c)$-space of the two examples given above is in order (see Fig. 3). In both cases the $\dot{k}=0$ curve breaks up in two parts. The first part, with steady state $k_{1}^{*}$ is very similar to the standard neoclassical growth model. The second part, with steady states $k_{2}^{*}$ and $k_{3}^{*}$, looks like an island and is split in two parts by the $1+k g_{c}(c)=0$ locus. ${ }^{11}$ At the steady states the $\dot{c}=0$ locus is locally vertical. The arrows indicating the direction of change in $c$ and $k$ illustrate the saddle point stability of steady states $k_{1}^{*}$ and $k_{3}^{*}$ and the instability of $k_{2}^{*}$.

The poverty trap is clearly illustrated in Fig. 3 as there is a range of capitallabor ratios such that $\dot{k}<0$, independent of the consumption level chosen. The only viable option for poor countries is to put the economy on the saddle path to low income steady state $k_{1}^{*}$.

An economy starting with an initial capital-labor ratio $k(0)$ in between points $\boldsymbol{A}$ and $\boldsymbol{B}$ in Fig. 4 has the option, in principle, to choose between the low income steady state $k_{1}^{*}$ and the high income steady state $k_{3}^{*}$. One cannot conclude that such an economy will choose the path leading to the high income steady state $k_{3}^{*}$. This is illustrated in Fig. 4, see also Blomqvist and Pitchford (1977) and Skiba (1978). The three fat dots represent steady states at $k_{1}^{*}, k_{2}^{*}$ and $k_{3}^{*}$. The spirals are the saddlepaths to steady states $k_{1}^{*}$ and $k_{3}^{*}$ that do not violate constraints on state, co-state and control variables. The relevant portions of the spirals are from points $\boldsymbol{A}$ and $\boldsymbol{B}$; otherwise it would be optimal to cycle on the spirals which would require jumps in $c$ (and hence $\lambda$ ) which would violate the necessary continuity in time.

Suppose that the initial capital-labor ratio is in between the boundaries represented by points $\boldsymbol{A}$ and $\boldsymbol{B}$ in Fig. 4 . There are two options available to the economy that do not violate the necessary conditions (with concomitant utility payoff $V_{i}=\int_{0}^{\infty} \exp (-\varrho t) U\left[c_{i}(t)\right] \mathrm{d} t$ for $\left.i=1,2\right)$ :

1) the economy can follow the saddle path to $k_{1}^{*}$, or

2) the economy can follow the saddlepath to $k_{3}^{*}$.

It is clear that the saddle path leading to steady state $k_{1}^{*}$ allows the economy to 'eat' some of its capital and therefore enables larger consumption levels initially. The saddle path leading to $k_{3}^{*}$, on the other hand, requires positive investments, hence forces the economy to consume less initially, for which it will be rewarded later on because it can ultimately reach higher consumption levels. Whether the economy prefers the saddle path to $k_{1}^{*}$ to the saddle path to $k_{3}^{*}$, then, depends on its impatience, measured by the rate of time preference $\varrho$. The larger $\varrho$, the more impatient the agents are, the more important the initially high level of consumption of the saddle path to $k_{1}^{*}$ becomes, ceteris paribus, ${ }^{12}$ hence the higher the value of $V_{1}-V_{2}$. Crudely speaking, therefore, for initial capital-labor ratios in between $\boldsymbol{A}$ and $\boldsymbol{B}$ ('middle income countries'), impatient countries will choose

11 This gives another justification for restricting attention to $(k, c) \in \mathscr{D}$, because for the same value of $k$ and $k$ (illustrated for $k=0$ in Fig. 3) the economy will always choose the highest level of $c$.

12 The ceteris paribus clause must be made here as changes in $\varrho$ will affect both the steady states and the paths to these steady states. 
the path to the low income steady state $k_{1}^{*}$, while patient countries will choose the path to the high income steady state $k_{3}^{*}$. Given the rate of time preference $\varrho$, however, there is a unique cut-off point $k_{T}$ in between points $\boldsymbol{A}$ and $\boldsymbol{B}$, as mentioned in Sect. 4, such that for all $k(0)<k_{T}$ the optimal trajectory is the one leading to the low income steady state, while for all $k(0)$ exceeding this 'trap' level the optimal trajectory is the one leading to the high income steady state.

\section{Conclusions}

Endogenous population growth, i.e. making the rate of population growth dependent on society's opulence (measured by consumption, production or the wage rate), causes parametric changes, such as changes in the rate of time preference or the depreciation rate, to have a larger impact on the steady state lavels of per capita consumption and capital. The modified neoclassical 'golden rule' of accumulation must be extended to take the dependence of the population growth rate on the per capita level of consumption into consideration. The neoclassical dichotomy, in which the rate of time preference, the depreciation rate and the production function dictate the steady state level of capital independently of the consumption level, then disappears.

More importantly, however, is the possible occurrence of multiple steady states, without resorting to irregularities or externalities in the production function. The steady states alternate in saddle point stability and make the 'poverty trap' a reality. The two main causes of multiplicity dubbed 'opulence sensitivity' and 'production sensitivity', are identified and illustrated by means of an example. Poor countries have initial levels of production and capital so low that they are forced to the low income steady state. Only a large injection of capital from abroad in the form of foreign aid might enable a poor country to reach the high income steady state.

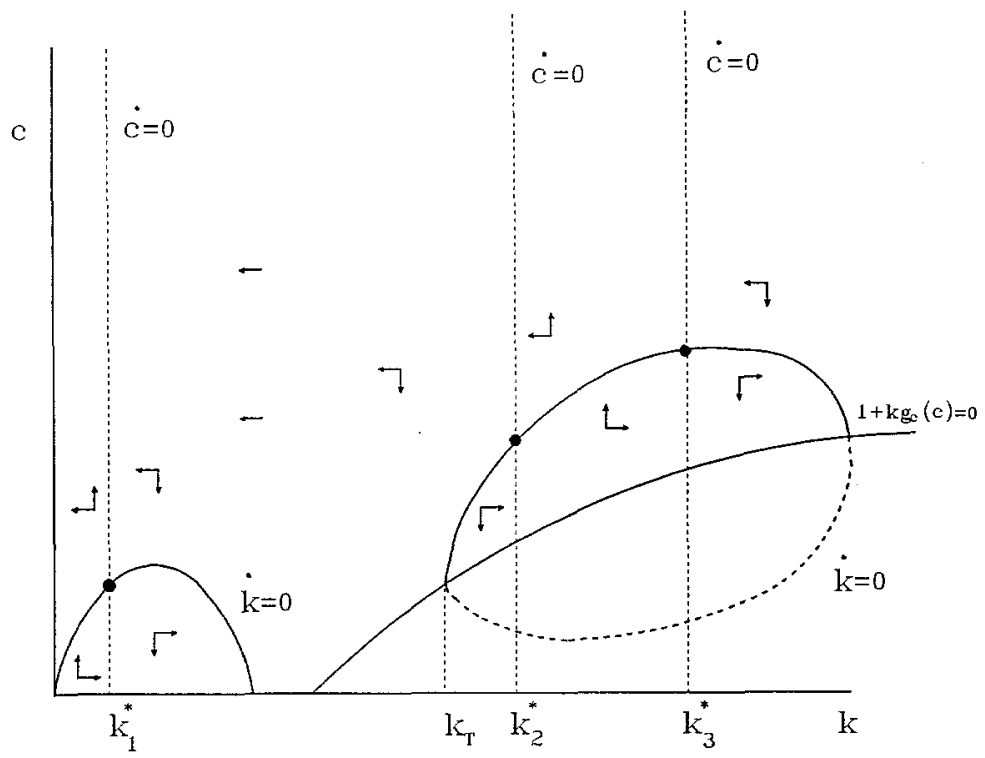

Fig. 3. Dynamics in $(k, c)$-space 


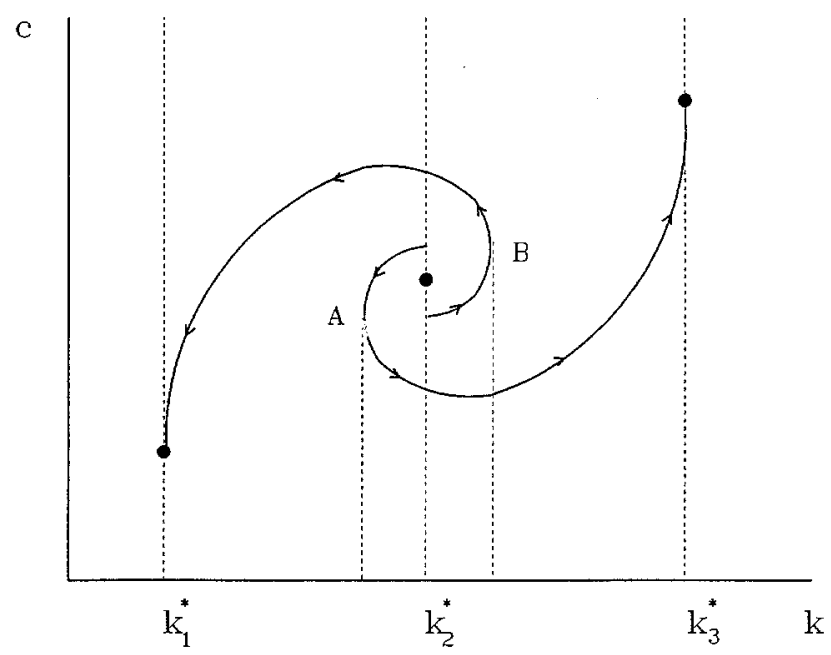

Fig. 4. Saddle path trajectories to $k_{1}^{*}$ and $k_{3}^{*}$

\section{Appendix}

This appendix shortly states the main results for production or wage driven population growth. The assumptions are $A 1, A 2, A 3^{\prime}$ and, instead of $A 4$, $A 4^{\prime} \lim _{k \rightarrow \infty}-k g_{k}(k)<\mu$.

The problem becomes

Problem $P^{\prime}$

$$
\begin{aligned}
& \max _{c} \int_{0}^{\infty} \exp (-\varrho t) U[c(t)] \mathrm{d} t \quad \text { subject to } \\
& \dot{k}(t)=f[k(t)]-\{\mu+g[k(t)]\} k(t)-c(t) \equiv q[k(t)]-c(t) \\
& k(0) \text { given. }
\end{aligned}
$$

Proposition 1'. Under assumptions $A 1, A 2, A 3^{\prime}$ and $A 4^{\prime}$ there exists a solution to problem $P$.

Proof. The only change in the proof of Proposition 1 is:

$$
\mathscr{F}_{k}=-U_{c}(c)<0 \quad \mathscr{F}_{k k}=-U_{c c}(c)<0 .
$$

Necessary conditions for optimality, derived from the Hamiltonian, are

$$
\begin{aligned}
& U_{c}(c)-\lambda=0 \\
& \dot{\lambda}=\lambda\left[\varrho-q_{k}(k)\right] \\
& \lim _{t \rightarrow \infty} k(t) \lambda(t) \exp (-\varrho t)=0 .
\end{aligned}
$$


Rewriting in terms of $c$ and $k$ gives

$$
\begin{aligned}
& \dot{c}=\left\{c / \sigma_{U_{c}}(c)\right\}\left[q_{k}(k)-\varrho\right] \\
& \dot{k}=q(k)-c .
\end{aligned}
$$

Steady state values $c^{*}$ and $k^{*}$ are given by

$$
\begin{aligned}
& q_{k}\left(k^{*}\right)-\varrho=0 \\
& q\left(k^{*}\right)-c^{*}=0
\end{aligned}
$$

The assumptions guarantee that there is at least one steady state. Multiplicity is possible because $q_{k k}(k)$ can change sign. It is straightforward to show that these steady states alternate in local saddlepoint stability. The $\dot{c}=0$ loci are given by vertical straight lines, while the shape of the $\dot{k}=0$ locus will depend on the specific properties of the $q(k)$ function.

\section{References}

Barro RJ, Becker GS (1989) Fertility choice in a model of economic growth. Econometrica $57: 481-501$

Benhabib J, Jovanovic B (1991) Externalities and growth accounting. Am Econ Rev 81:82-113

Blanchard OJ, Fischer S (1989) Lectures on macroeconomics. MIT Press, Cambridge, MA

Blomqvist AG, Pitchford JD (1977) Population policy, individual welfare and optimal economic growth. Mimeo Australian National University

Brock WA, Malliaris AG (1989) Differential equations, stability and chaos in dynamic economics. North-Holland, Amsterdam

Cigno A (1992) Children and pensions. Presidential address to the European Society for Population Economics, Pisa. J Popul Econ 5:175-183

Dechert WD, Nishimura K (1983) A complete characterization of optimal growth paths in an aggregated model with a non-concave production function. J Econ Theory 31:332-354

Dellas H, de Vries CG (1991) Piecemeal versus precipitous factor market integration. University of Leuven, CES discussion paper no. 82

Ehrlich I (1990) The problem of development: Introduction. J Polit Econ 98:s1-s11

Liviatan N, Samuelson PA (1969) Notes on turnpikes: stable and unstable. J Econ Theory 1:454-475

Lucas RE Jr (1988) On the mechanics of economic development. J Mon Econ 22:3-42

Mankiw NG, Romer D, Weil DN (1992) A contribution to the empirics of economic growth. Q J Econ 107:407-437

Nelson RR (1956) A theory of the low level equilibrium trap in underdeveloped economies. Am Econ Rev 46:894-908

Pitchford JD (1974) Population in economic growth. North-Holland, Amsterdam

Raut LK (1991) Capital accumulation, income distribution and endogenous fertility in an overlapping generations general equilibrium model. J Dev Econ 34:123-150

Romer PM (1986a) Increasing returns and long-run growth. J Polit Econ 94:1002-1037

Romer PM (1986b) Cake eating, chattering, and jumps: Existence results for variational problems. Econometrica 54:897-908

Rosenzweig MR (1990) Population growth and human capital investments: Theory and evidence. J Polit Econ 98:s 38-s70

Sato R, Davis EG (1971) Optimal savings policy when labor grows endogenously. Econometrica $39: 877-897$

Skiba AK (1978) Optimal growth with a convex-concave production function. Econometrica 46:527-539

Unites Nations (1983) Statistical Yearbook

World Bank (1987) World development report 